\title{
Microstructural Evolution of Chessboard like Nanodomains in Mn-doped $\mathrm{ZnGaO}_{4} \mathrm{Spinel}$
}

Avnish Pal ${ }^{1}$, Aman KumarLal Das ${ }^{2}$, Manish Singh ${ }^{3}$, Chanchal Ghosh ${ }^{3}$, Paul Kotula ${ }^{4}$, C. Barry Carter ${ }^{3}$ and Joysurya Basu ${ }^{5}$

${ }^{1}$ Department of Metallurgical Engineering IIT (BHU), Varanasi, Uttar Pradesh, India, ${ }^{2}$ Department of Metallurgical Engineering, Indian Institute of Technology (BHU), UP, India, United States, ${ }^{3}$ University of Connecticut, Connecticut, United States, ${ }^{4}$ Sandia National Laboratories, Albuquerque, New Mexico, United States, ${ }^{5}$ Department of Metallurgical Engineering, IIT (BHU), Varanasi, Uttar Pradesh, India

Well-organized self-assembled chessboard (CB) nanostructures have several potential applications including as high density recording medium, nanoscale heat pumps, and so on. Mn-doped $\mathrm{ZnGaO} 4$, in particular, is a potential candidate for low-dimensional heat pumps. (Ni \& Khachaturyan, 2008; Ni \& Khachaturyan, 2009). Since, each chessboard-like microstructure consists of two identical and periodically distributed nanodomains possessing two distinct properties, like thermoelectric-non thermoelectric in case of $\mathrm{ZnGaMnO} 4$, this may facilitate the construction of perpendicular magnetic recording media with enhanced storage density. To convert this concept into realization elucidation of growth mechanism is required to optimize the growth parameters.

In the present study, Mn-doped $\mathrm{ZnGaO} 4$ samples were prepared by high temperature solid-state reaction technique. Samples were investigated for their microstructural evolutions, phase transformations and chemistry. The evolution of CB nanostructures has been observed at various intermediate stages. In order to study these intermediate stages, samples at different stages of growth have been extensively investigated with various TEM techniques, such as diffraction contrast imaging, HAADF-STEM, CBED, and HRTEM coupled with image simulations (Williams \& Carter, 2009). The comprehensive investigation of interfaces of nanodomains has provided significant information about this phase separation event, which shows that the whole microstructural evolution is a multistage process whose first step is twinning of domains, in which cubic parent phase turn into twin related metastable tetragonal phase with minimal c/a ratio. These metastable tetragonal domains again separated compositionally into cubic and tetragonal phases over the time. Tetragonality of the Mn-rich domain has been increased over the time and simultaneously alternative domain became rich in Ga with increased cubicity. Hence, chessboard systems possess coherent twinned interfaces which are compositionally distinguishable; phase separation is the final stage of this microstructural evolution. Systematic tilting along appropriate zone axes excites various microstructural features in diffraction contrast imaging mode. They include contrast in nanodomain along a specific zone axis, fringes in dark domains, simultaneous herringbone fringes in both the nanodomains, and cuboidal shape of nanodomains. Dark domains display random fringe-like contrast prominently because these particular domains are satisfying the Bragg diffraction condition whereas preferentially oriented fringes within the domains are basically subdomains laying inside with respect to parent domain. These subdomains are gone out of Bragg's diffraction condition that is why they are appearing brighter. These parent and product phases are oriented in such a manner so that they could minimize the interfacial energy, in turn reducing the free energy of the whole system.

Figure 1 shows a representative BF-TEM micrograph and corresponding selected area electron diffraction pattern from $\mathrm{Mn}$-doped $\mathrm{ZnGaO} 4$. Presence of bright and dark features have been observed due to varying diffraction conditions (cf. Fig. 1a) and SAD pattern could be indexed to those of cubic phase along $<$ ![if !msEquation] $><$ ! [if !vml] $>\quad<$ ![endif] $><$ ![endif] $>$ zone axis (Fig. 1b).

HAADF-STEM and nanobeam electron diffraction techniques were used for compositional mapping and crystal-structure identification of these nanodomains. Mapping along [010] direction of crystal has given a lamella like appearance in the STEM mode ratified that one domain is Mn rich and another is Mn lean. Nano 
diffraction patterns corresponding to $\mathrm{Mn}$ rich, and Mn lean domains have shown that the Mn-rich domain is tetragonal with a c/a ratio of 1.09 and the Ga-rich domain is cubic. However, HAADF-STEM mapping has given some elementary insight about compositional distribution but as diffraction contrast images inferring the 3-D complex morphology of the CB. All of these results will be included during this presentation.

Part of the investigation was carried out at CINT, an Office of Science User Facility operated for the U.S. DOE, and in the Materials Characterization Department. Sandia National Labs is managed and operated by National Technology and Engineering Solutions of Sandia, LLC., a wholly owned subsidiary of Honeywell International, Inc., for the U.S. DOE's NNSA contract DE-NA-0003525. The views expressed here do not necessarily represent the views of the U.S. DOE or the U.S. Government.

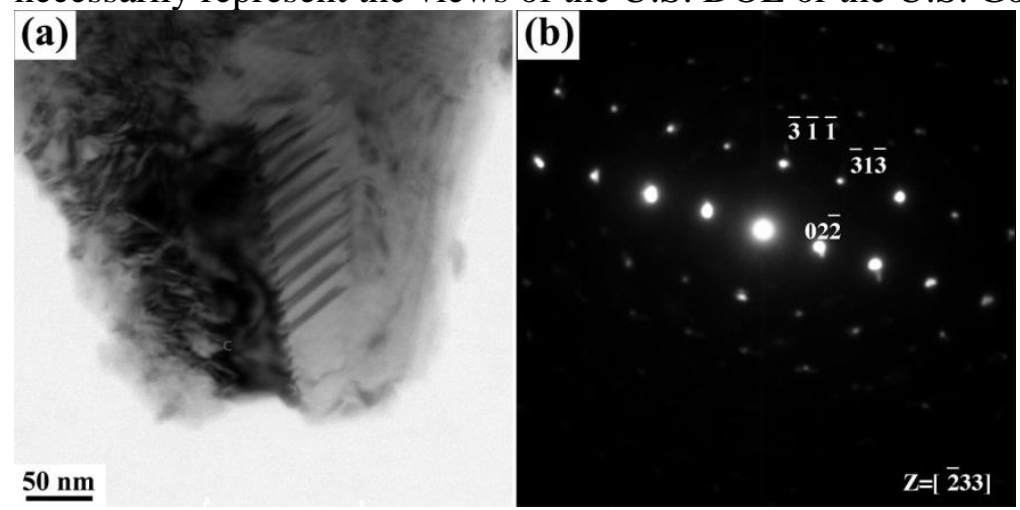

Figure 1. (a) Bright-field TEM micrograph and (b) corresponding selected-area electron-diffraction pattern from $\mathrm{Mn}$-doped $\mathrm{ZnGaO} 4$.

\section{References}

Ni, Y.\& KhaCHATURYAN, A.G. (2008). Mechanism and conditions of the chessboard structure formation. Acta Mater 56(16), 4498-4509.

Ni, Y. \& Khachaturyan, A.G. (2009). From chessboard tweed to chessboard nanowire structure during pseudospinodal decomposition. Nature materials 8(5), 410-414.

Williams, D.B. \& CARTER, C.B. (2009). Transmission Electron Microscopy. Part 3: Imaging. Springer, New York. 